https://helda.helsinki.fi

\title{
Thouless-Valatin rotational moment of inertia from linear response theory
}

\section{Petrik, Kristian}

2018-03-22

Petrik , K \& Kortelainen , M 2018 , ' Thouless-Valatin rotational moment of inertia from linear response theory ' , Physical Review C , vol. 97 , no. 3 , 034321 . https://doi.org/10.1103/PhysRevC.97.034321

http://hdl.handle.net/10138/235447

https://doi.org/10.1103/PhysRevC.97.034321

cc_by

publishedVersion

Downloaded from Helda, University of Helsinki institutional repository.

This is an electronic reprint of the original article.

This reprint may differ from the original in pagination and typographic detail.

Please cite the original version. 


\title{
Thouless-Valatin rotational moment of inertia from linear response theory
}

\author{
Kristian Petrík* and Markus Kortelainen ${ }^{\dagger}$ \\ Helsinki Institute of Physics, University of Helsinki, P.O. Box 64, FI-40014 Helsinki, Finland \\ and Department of Physics, University of Jyvaskyla, P.O. Box 35, FI-40014 Jyvaskyla, Finland
}

(Received 25 September 2017; published 22 March 2018)

\begin{abstract}
Spontaneous breaking of continuous symmetries of a nuclear many-body system results in the appearance of zero-energy restoration modes. These so-called spurious Nambu-Goldstone modes represent a special case of collective motion and are sources of important information about the Thouless-Valatin inertia. The main purpose of this work is to study the Thouless-Valatin rotational moment of inertia as extracted from the Nambu-Goldstone restoration mode that results from the zero-frequency response to the total-angular-momentum operator. We examine the role and effects of the pairing correlations on the rotational characteristics of heavy deformed nuclei in order to extend our understanding of superfluidity in general. We use the finite-amplitude method of the quasiparticle random-phase approximation on top of the Skyrme energy density functional framework with the Hartree-Fock-Bogoliubov theory. We have successfully extended this formalism and established a practical method for extracting the Thouless-Valatin rotational moment of inertia from the strength function calculated in the symmetry-restoration regime. Our results reveal the relation between the pairing correlations and the moment of inertia of axially deformed nuclei of rare-earth and actinide regions of the nuclear chart. We have also demonstrated the feasibility of the method for obtaining the moment of inertia for collective Hamiltonian models. We conclude that from the numerical and theoretical perspective, the finite-amplitude method can be widely used to effectively study rotational properties of deformed nuclei within modern density functional approaches.
\end{abstract}

DOI: 10.1103/PhysRevC.97.034321

\section{INTRODUCTION}

Collective modes or excitations of many-fermion systems are excellent sources of vital information about the properties of the effective interaction among the constituent particles and correlations that govern the dynamics of the many-body system. It is a major objective for the nuclear theory to extensively describe all types of collective nuclear motion. Studying the low-lying excitations brings better understanding of the properties of pairing correlations, shell structure or nuclear deformation, while the higher-lying giant nuclear resonances reveal essential details about nuclear photoabsorption reactions or nuclear matter symmetry energy and compressibility [1-4]. One can analyze a response of a nucleus to a time-dependent external field to gain important knowledge about such excited states.

There exist several groups of models dealing with the many-body structure and low-energy dynamics of atomic nucleus; for example, interacting shell-model approaches, $a b$ initio methods, or the density functional theory (DFT) formalism. The nuclear DFT, which belongs to the family of the self-consistent mean-field (SCMF) approaches, determines the nuclear one-body mean field by using an energy density functional (EDF) adjusted for a given task [5]. Within the SCMF approach, the nuclear mean-field is obtained by solving the Hartree-Fock (HF) or Hartree-Fock-Bogoliubov (HFB)

\footnotetext{
*kristian.k.petrik@jyu.fi

${ }^{\dagger}$ markus.kortelainen@jyu.fi
}

equations self-consistently. Currently, the nuclear DFT can rather successfully describe the nuclear ground-state properties throughout the whole nuclear chart [6-8].

An important ingredient of the nuclear DFT approach is the concept of a spontaneous symmetry breaking. A meanfield wave function, obtained self-consistently, usually breaks some of the symmetries of the full Hamiltonian. In principle, an exact ground-state wave function of a nucleus does not break symmetries of the underlying many-body Hamiltonian; however, such a type of wave-function is often computationally out of reach and simplifying approximations have to be used. Effectively, the spontaneous symmetry breaking allows us to introduce diverse short-range and long-range correlations to the deformed wave function.

To access various excitation modes, one needs to go beyond the static mean field. One of the most utilized methods is the linear-response theory; that is, the random-phase approximation (RPA) theory. Excellent progress has been achieved with the application of the RPA and the quasiparticle random-phase approximation (QRPA), the superfluid extension of the RPA, to the excited collective states of nuclei within the nuclear DFT framework. Modern approaches usually employ the selfconsistent RPA or QRPA calculations together with wellestablished EDFs [5,9-12]. However, traditional approaches, such as the very successful matrix formulation of the quasiparticle random-phase approximation (MQRPA), suffer from the fact that they are computationally heavy, especially when spherical symmetry is broken. This is the main reason why the QRPA formalism has been able to treat the deformed nuclei only recently. 
To deal with various drawbacks of standard methods for collective excitations, a very efficient formalism has been proposed. The so-called finite amplitude method (FAM) was introduced, first to calculate the RPA strength functions [13] and later extended to spherically symmetric QRPA (FAMQRPA) [14]. The FAM-QRPA promptly became a powerful tool to effectively handle a broad range of nuclear phenomena [15]. For example, it was successfully applied to study deformed axially symmetric nuclear systems within the HFB-Skyrme framework, individual QRPA modes, beta-decay modes, collective moments of inertia, collective dynamics, quadrupole and octupole strengths, or the giant dipole resonance (GDR) [16-23]. Due to its many distinct advantages, we chose the FAM-QRPA as the main theoretical framework for the purposes of this work.

The properties and dynamics of heavy nuclear systems can provide helpful insights for the physics of confined atomic fermions, especially when superfluid characteristics are of interest [24-30]. Although the length and energy scales of confined atomic systems are quite different from those of nuclear scale, the rotating superfluid Fermi-liquid-drop picture of a nucleus covers the essential concepts to build up a connection between these two fields. One of the connecting ingredients is the moment of inertia, which is believed to provide an unambiguous signature of superfluidity in general. Since the similarities between nuclear systems and trapped fermions are strong, studying the rotational moment of inertia of heavy deformed nuclei by the best available theoretical methods will offer crucial information about the phenomenon of superfluidity. This paper is thus aimed to present results that will be valuable not only for the nuclear physics, but for the physics of trapped fermionic gases. Such universality can also help to study the transition between macroscopic and microscopic fermionic systems.

It is well known that the experimental moments of inertia of nuclei are usually notably smaller than the corresponding rigid-body values [31]. When deriving the expression for the moment of inertia, the simplest case leads to the Inglis moment of inertia, which represents the response of free gas. The moment of inertia resulting from this basic Inglis formula is typically very close to the rigid-body values. To improve the description of the data, one has to take into account the pairing correlations, which lead, within the BCS formalism, to the so-called Belyaev formula. This superfluid expansion of the Inglis inertia lowers the theoretical values towards the experimental results, which manifests the importance of the correlations of the pairing type.

More generally, one can obtain the moment of inertia from the self-consistent cranking theory or the linear-response theory as derived by Thouless and Valatin [32]. In this context, the inertia also takes into account the nuclear response, i.e., the effects of induced fields that represent a reaction of the system to an external perturbation, determined by a given operator. In this way, the moment of inertia is defined by a generalized expression that contains, as special cases, both Inglis and Belyaev formulas.

Although the moment of inertia of superfluid nuclei is significantly smaller than the rigid-body inertia, it is still larger than that of a strictly irrotational motion. From this observation, we can conclude that the currents in superfluid rotating nuclei have a two-component character: the total current consists of rotational and irrotational components and the moment of inertia is the key observable to determine the effects of pairing correlations on the collective motion of nuclear systems. Analogous behavior is found to be present in the trapped superfluid Fermi gases at zero and nonzero temperatures, where the temperature dependence is shown to have crucial effects on the moment of inertia as well as the qualitative behavior of the currents $[25,26]$.

The main objective of this work is to apply the FAMQRPA approach to study the Thouless-Valatin (TV) rotational moment of inertia in various nuclear systems and inspect the significance of the pairing correlations with respect to the rotational characteristics of studied nuclei. The TV inertia can be obtained from the spurious zero-frequency (zero-energy) mode, which is a consequence of a broken symmetry. We propose an efficient and numerically accessible method for extracting this quantity from the total-angular-momentum operator that acts as an external perturbation. This method can be easily used to describe collective modes of a wide variety of deformed nuclei.

As a proof of concept, we also demonstrate the usefulness of our method in providing local QRPA calculations, specifically constrained calculations of the rotational moment of inertia as a function of density. In this way, FAM-QRPA could be used to easily access collective mass parameters that represent a key input for microscopic collective Hamiltonian models.

Our paper is organized in the following way: In Sec. II, an overview of the theoretical framework is presented, in which the time-dependent HFB theory and FAM-QRPA approach are discussed in detail. Additionally, we briefly analyze the spontaneous-symmetry-breaking phenomenon and its connection to the collective TV inertia. In Sec. III, the numerical setup and main FAM-QRPA results are covered. Final conclusions and prospects are given in Sec. IV.

\section{THEORETICAL FRAMEWORK}

In this section we recapitulate all necessary theoretical details and go through the essential expressions that were used as a foundation for our calculations. The main ideas and assets of the FAM-QRPA are discussed.

\section{A. Finite-amplitude method}

A static mean-field approach without pairing correlations (Hartree-Fock) or with pairing correlations (Hartree-FockBogoliubov) can basically provide the nuclear binding energy and other ground-state bulk properties. To access excited states and dynamics of the system, one has to go beyond the meanfield formalism. One approach is to apply time-dependent extensions to the stationary mean-field models. In the following, we use the quasiparticle random-phase approximation as a theoretical method that represents a small-amplitude limit of the time-dependent superfluid HFB (TD-HFB) theory.

The HFB ground state $|\Phi\rangle$ is obtained by the minimization of the total energy defined through the energy density $\mathcal{E}\left(\rho, \kappa, \kappa^{*}\right)$. In general, expectation values of an operator with 
an HFB state can be expressed with one-body densities. In the quasiparticle picture, the energy density $\mathcal{E}\left(\rho, \kappa, \kappa^{*}\right)$ introduces the one-body density matrix $\rho$ and the pairing tensor $\kappa$,

$$
\begin{aligned}
& \rho_{i j}=\left\langle\Phi\left|\hat{c}_{j}^{\dagger} \hat{c}_{i}\right| \Phi\right\rangle=\left(V^{*} V^{T}\right)_{i j}=\rho_{j i}^{*}, \\
& \kappa_{i j}=\left\langle\Phi\left|\hat{c}_{j} \hat{c}_{i}\right| \Phi\right\rangle=\left(V^{*} U^{T}\right)_{i j}=-\kappa_{j i},
\end{aligned}
$$

where $V$ and $U$ are the Bogoliubov transformation matrices that define the linear relation between the Bogoliubov quasiparticles $\hat{a}_{\mu}^{\dagger}, \hat{a}_{\mu}$ and the bare particles $\hat{c}_{k}^{\dagger}, \hat{c}_{k}$,

$$
\begin{aligned}
& \hat{a}_{\mu}^{\dagger}=\sum_{k}\left[U_{k \mu} \hat{c}_{k}^{\dagger}+V_{k \mu} \hat{c}_{k}\right], \\
& \hat{a}_{\mu}=\sum_{k}\left[U_{k \mu}^{*} \hat{c}_{k}^{\dagger}+V_{k \mu}^{*} \hat{c}_{k}\right] .
\end{aligned}
$$

The single-particle Hamiltonian $h$ and the pairing potential $\Delta$ follow from the variation of the energy density functional $\mathcal{E}$ with respect to $\rho$ and $\kappa^{*}$,

$$
h_{i j}\left(\rho, \kappa, \kappa^{*}\right)=\frac{\partial \mathcal{E}}{\partial \rho_{i j}}, \quad \Delta_{i j}\left(\rho, \kappa, \kappa^{*}\right)=\frac{\partial \mathcal{E}}{\partial \kappa_{i j}^{*}} .
$$

By minimizing the total Routhian, one can derive the HFB equations in terms of the transformation matrices $V$ and $U$,

$$
\left(\begin{array}{cc}
h-\lambda & \Delta \\
-\Delta^{*} & -h^{*}+\lambda
\end{array}\right)\left(\begin{array}{c}
U_{\mu} \\
V_{\mu}
\end{array}\right)=E_{\mu}\left(\begin{array}{c}
U_{\mu} \\
V_{\mu}
\end{array}\right),
$$

where $E_{\mu}$ are the quasiparticle energies and $\lambda$ is the chemical potential introduced in order to fix the average particle number.

The TD-HFB can be used to describe the time evolution of the quasiparticles under a one-body external perturbation that induces a polarization on the HFB ground state. Such a perturbation can be expressed as a time-dependent field in a conjugate form,

$$
\hat{F}(t)=\eta\left[\hat{F}(\omega) e^{-i \omega t}+\hat{F}^{\dagger}(\omega) e^{i \omega t}\right],
$$

where $\eta$ is a small real parameter introduced for the purpose of the small amplitude approximation, used in the FAM-QRPA.

The time evolution of a quasiparticle operator under the influence of the external field that forces the oscillations can be expressed as the TD-HFB equation,

$$
i \hbar \frac{\partial}{\partial t} \hat{a}_{\mu}(t)=\left[\hat{H}(t)+\hat{F}(t), \hat{a}_{\mu}(t)\right],
$$

where the quasiparticle oscillations are given as

$$
\begin{aligned}
\hat{a}_{\mu}(t) & =\left[\hat{a}_{\mu}+\delta \hat{a}_{\mu}(t)\right] e^{i E_{\mu} t}, \\
\delta \hat{a}_{\mu}(t) & =\eta \sum_{\nu} \hat{a}_{\nu}^{\dagger}\left[X_{\nu \mu}(\omega) e^{-i \omega t}+Y_{\nu \mu}^{*}(\omega) e^{i \omega t}\right],
\end{aligned}
$$

where $X_{v \mu}$ and $Y_{\nu \mu}$ are the FAM-QRPA amplitudes and $E_{\mu}$ is the one-quasiparticle energy.

The time-independent part $\hat{F}(\omega)$ of the one-body external perturbation $\hat{F}(t)$ can be now expressed (under the linear approximation) in the quasiparticle space as

$$
\hat{F}(\omega)=\sum_{\mu<\nu}\left[F_{\mu \nu}^{20}(\omega) \hat{a}_{\mu}^{\dagger} \hat{a}_{\nu}^{\dagger}+\hat{F}_{\mu \nu}^{02}(\omega) \hat{a}_{\mu} \hat{a}_{\nu}\right] .
$$

The external perturbation induces oscillations of the density atop the static HFB solution, thus the self-consistent Hamiltonian will also contain the induced part as $\hat{H}(t)=$ $\hat{H}_{\mathrm{HFB}}+\delta \hat{H}(t)$, where the oscillating part $\delta \hat{H}(t)$ is defined in a similar way as the external field in Eq. (5).

The response of the self-consistent Hamiltonian, given by the induced time-independent matrices $\delta H_{\mu \nu}^{20}$ and $\delta H_{\mu \nu}^{02}$, can be expressed with the HFB matrices $U$ and $V$ and induced fields $\delta h, \delta \Delta$, and $\overline{\delta \Delta}$ as

$$
\begin{aligned}
\delta H_{\mu \nu}^{20}(\omega)= & {\left[U^{\dagger} \delta h(\omega) V^{*}-V^{\dagger} \delta h(\omega)^{T} U^{*}\right.} \\
& \left.-V^{\dagger} \overline{\delta \Delta}(\omega)^{*} V^{*}+U^{\dagger} \delta \Delta(\omega) U^{*}\right]_{\mu \nu}, \\
\delta H_{\mu \nu}^{02}(\omega)= & {\left[U^{T} \delta h(\omega)^{T} V-V^{T} \delta h(\omega) U\right.} \\
& \left.-V^{T} \delta \Delta(\omega) V+U^{T} \overline{\delta \Delta}(\omega)^{*} U\right]_{\mu \nu} .
\end{aligned}
$$

Here, the fields $\delta h, \delta \Delta$, and $\overline{\delta \Delta}$ were obtained through the explicit linearization of the Hamiltonian ( $\eta$ parameter thus cancels out) and can therefore be expressed by using fields linearized with respect to perturbed densities; namely, $h^{\prime}$ and $\Delta^{\prime}$ :

$$
\begin{aligned}
& \delta h(\omega)=h^{\prime}\left[\rho_{\mathrm{f}}, \kappa_{\mathrm{f}}, \bar{\kappa}_{\mathrm{f}}\right], \\
& \delta \Delta(\omega)=\Delta^{\prime}\left[\rho_{\mathrm{f}}, \kappa_{\mathrm{f}}\right], \\
& \overline{\delta \Delta(\omega)}=\Delta^{\prime}\left[\bar{\rho}_{\mathrm{f}}, \bar{\kappa}_{\mathrm{f}}\right],
\end{aligned}
$$

where the non-Hermitian density matrices depend on the external perturbation through the FAM-QRPA amplitudes,

$$
\begin{aligned}
& \rho_{\mathrm{f}}(\omega)=+U X(\omega) V^{T}+V^{*} Y(\omega)^{T} U^{\dagger}, \\
& \bar{\rho}_{\mathrm{f}}(\omega)=+V^{*} X(\omega)^{\dagger} U^{\dagger}+U Y(\omega)^{*} V^{T}, \\
& \kappa_{\mathrm{f}}(\omega)=-U X(\omega)^{T} U^{T}-V^{*} Y(\omega) V^{\dagger}, \\
& \bar{\kappa}_{\mathrm{f}}(\omega)=-V^{*} X(\omega)^{*} V^{\dagger}-U Y(\omega)^{\dagger} U^{T} .
\end{aligned}
$$

To access a transition strength function one needs to obtain the FAM-QRPA amplitudes $X_{\mu \nu}(\omega)$ and $Y_{\mu \nu}(\omega)$. By using the linear approximation (i.e., linear response) one can derive the FAM-QRPA equations, which are given as

$$
\begin{aligned}
X_{\mu \nu}(\omega) & =-\frac{\delta H_{\mu \nu}^{20}(\omega)-F_{\mu \nu}^{20}(\omega)}{E_{\mu}+E_{\nu}-\omega}, \\
Y_{\mu \nu}(\omega) & =-\frac{\delta H_{\mu \nu}^{02}(\omega)-F_{\mu \nu}^{02}(\omega)}{E_{\mu}+E_{\nu}+\omega} .
\end{aligned}
$$

Since induced matrices $\delta H_{\mu \nu}^{20}$ and $\delta H_{\mu \nu}^{02}$ depend on the $X_{\mu \nu}$ and $Y_{\mu \nu}$ amplitudes, one has to employ an iterative, self-consistent scheme to solve the FAM-QRPA equations.

Formally, the FAM-QRPA system (12) is equivalent to the linear-response theory,

$$
R(\omega)^{-1}\left(\begin{array}{l}
X(\omega) \\
Y(\omega)
\end{array}\right)=-\left(\begin{array}{l}
F^{20} \\
F^{02}
\end{array}\right),
$$

where the response function $R(\omega)$ can be expressed as

$$
R(\omega)^{-1}=\left[\left(\begin{array}{cc}
A & B \\
B^{*} & A^{*}
\end{array}\right)-\omega\left(\begin{array}{cc}
1 & 0 \\
0 & -1
\end{array}\right)\right]
$$

where $A$ and $B$ are the well-known QRPA matrices. If the external field is set to zero, the linear-response equation (13) 
transforms to the standard matrix QRPA equation

$$
\left(\begin{array}{cc}
A & B \\
B^{*} & A^{*}
\end{array}\right)\left(\begin{array}{l}
X(\omega) \\
Y(\omega)
\end{array}\right)=\omega\left(\begin{array}{c}
X(\omega) \\
-Y(\omega)
\end{array}\right) .
$$

Since matrices $A$ and $B$ typically have very large dimensions in the deformed case, solving the above equation is computationally rather demanding. The essential asset of the FAMQRPA lies in the fact that, instead of a very time-consuming construction and diagonalization of the large QRPA matrix within the standard MQRPA procedure, one calculates the FAM-QRPA amplitudes iteratively with respect to the response of the self-consistent Hamiltonian, i.e., induced fields $\delta H^{20}$ and $\delta H^{02}$, to the external field. This significantly reduces the computational cost in comparison with the MQRPA.

The transition strength function of the operator $\hat{F}$ at the frequency $\omega$ is defined as

$$
\frac{d B(\hat{F} ; \omega)}{d \omega}=-\frac{1}{\pi} \operatorname{Im} S(\hat{F} ; \omega),
$$

where the FAM-QRPA strength function $S(\hat{F} ; \omega)$ can be expressed as

$$
S(\hat{F} ; \omega)=\sum_{\mu<\nu}\left[F_{\mu \nu}^{20 *} X_{\mu \nu}(\omega)+F_{\mu \nu}^{02 *} Y_{\mu \nu}(\omega)\right] .
$$

To obtain a finite value for the FAM-QRPA transition strength, a small imaginary part, $\omega \rightarrow \omega_{\gamma}=\omega+i \gamma$, is added to the excitation energy. This leads to a Lorentzian smearing of transition strength function with a width of $\Gamma=2 \gamma$. However, in order to access a Nambu-Goldstone mode, one needs to evaluate strength function (17) at the vanishing frequency $\omega_{\gamma}=\omega=0$. We will discuss this relation in the next section.

\section{B. Collective Thouless-Valatin inertia and Nambu-Goldstone modes}

When some form of a mean-field approximation is introduced, a spontaneous-symmetry-breaking phenomenon that contains information about correlations to the one-body approximation can occur. Continuous symmetries conserved by the exact many-body Hamiltonian, which may be broken spontaneously, are the translational, rotational, and particle number gauge symmetry [1]. In addition, the isospin symmetry is broken explicitly by the presence of the Coulomb interaction and spontaneously due to the mean field [33].

As a result, spontaneous breaking of any of the above continuous symmetries leads to the appearance of a new zero-energy mode that restores the given symmetry. Such zeroenergy restoration modes are called Nambu-Goldstone (NG) modes and are a consequence of the symmetry-broken mean field and represent a special case of collective motion [1,34,35]. They are also associated with the infinitesimal transformation of the frame of reference and, since they do not represent real physical excitations in the intrinsic frame, they are often called spurious modes.

It is well known that breaking of the translational symmetry introduces the center of mass NG mode, rotational symmetry the rotational $\mathrm{NG}$ mode, and particle number gauge symmetry the pairing-rotational NG mode. In the intrinsic frame, each NG mode can be associated with a collective inertia that has experimental correspondence to an actual spectroscopy measurement in the laboratory frame. In the QRPA framework, such collective inertia coupled to the NG mode is called the Thouless-Valatin inertia [20,32]. For the translational (center of mass) NG mode the TV inertia is simply the total mass of the nucleus and is easily obtainable since the coordinate and momentum QRPA phonon operators, needed for the estimation, are known in advance. In the case of the (pairing-) rotational $\mathrm{NG}$ mode the $\mathrm{TV}$ inertia represents the nuclear (pairing-) rotational moment of inertia of the nucleus.

With the exception of the center-of-mass NG mode, in order to obtain the TV inertia one has to fully solve the QRPA equations. Although all necessary expressions are known in the standard matrix QRPA formalism, the full evaluation of the QRPA $A$ and $B$ matrices is computationally heavy, mostly due to their large dimensions. Other equivalent approaches have been therefore employed to obtain this quantity. The most important ones are the perturbation expansion procedure in the adiabatic time-dependent HFB theory, the cranked meanfield calculations within the HFB theory and, most recently, the FAM-QRPA approach developed for the nuclear density functional theory.

The FAM-QRPA has been already successfully formulated for calculating the symmetry-restoring NG modes of the translational and particle number gauge symmetries [20,36]. It has been shown that the TV inertia calculated in this way provides crucial information about the ground-state correlations to the relevant broken symmetry and that the FAM-QRPA represents a very precise and effective method to study this quantity. Our present work continues in this direction and extends the FAM-QRPA also to the case of the rotational NG mode and related rotational TV moment of inertia.

To obtain information about the given NG mode one can use a relevant one-body operator as the external field and evaluate the value of the strength function at zero frequency. In the case of translational symmetry, which introduces the center-of-mass NG mode, both the coordinate $\hat{Q}$ and momentum operators $\hat{P}$ are known and the TV inertia is simply the total mass of the studied nucleus, $M_{\mathrm{NG}}=A m$, where $A$ is the atomic mass number and $m$ is mass of the nucleon. This is the only example where it is not necessary to solve QRPA equations to obtain the TV inertia.

The aim of this work is to study the rotational ThoulessValatin moment of inertia of the rotational NG mode, where the one-body operator of interest is the total angular momentum. Depending on the symmetries chosen, a specific component $\hat{J}_{i}$ of the angular-momentum operator has to be selected. Such a component, when used as external perturbation, leads to the rotational TV inertia that can be extracted from the strength function at zero frequency as

$$
\begin{aligned}
S\left(\hat{J}_{i} ; 0\right)_{\mathrm{NG}} & =\sum_{\mu<\nu}\left[\left(J_{i}^{20 *}\right)_{\mu \nu} X_{\mu \nu}(0)+\left(J_{i}^{02 *}\right)_{\mu \nu} Y_{\mu \nu}(0)\right] \\
& =-M_{\mathrm{NG}}^{J_{i}} .
\end{aligned}
$$

Here, $M_{\mathrm{NG}}^{J_{i}}$ is the TV moment of inertia along the $i$ axis. To shortly recapitulate the derivation of Eq. (18), we can consider two conjugate operators, $\hat{P}$ and $\hat{Q}$, connected to the broken 
symmetry. At zero energy, the solution for the NG mode can be written [1], by using the QRPA matrices, as

$$
\begin{gathered}
\left(\begin{array}{cc}
A & B \\
B^{*} & A^{*}
\end{array}\right)\left(\begin{array}{c}
P^{20} \\
-P^{20 *}
\end{array}\right)=0 \\
\left(\begin{array}{cc}
A & B \\
B^{*} & A^{*}
\end{array}\right)\left(\begin{array}{c}
Q^{20} \\
-Q^{20 *}
\end{array}\right)=-\frac{i}{M_{\mathrm{NG}}}\left(\begin{array}{c}
P^{20} \\
P^{20 *}
\end{array}\right) .
\end{gathered}
$$

These equations can be solved to obtain the mass parameter for NG mode as

$$
\begin{aligned}
M_{\mathrm{NG}}= & 2\left[\operatorname{Re}\left(P^{20}\right)(A+B)^{-1} \operatorname{Re}\left(P^{20}\right)\right. \\
& \left.+\operatorname{Im}\left(P^{20}\right)(A-B)^{-1} \operatorname{Im}\left(P^{20}\right)\right] .
\end{aligned}
$$

Next, by using the linear-response equation (13) to connect the QRPA amplitudes with the QRPA matrices, the strength function (17) can be written as

$$
\begin{aligned}
S(\hat{P}, 0)= & -2 \operatorname{Re}\left(P^{20}\right)(A+B)^{-1} \operatorname{Re}\left(P^{20}\right) \\
& -2 \operatorname{Im}\left(P^{20}\right)(A-B)^{-1} \operatorname{Im}\left(P^{20}\right) \\
= & -M_{\mathrm{NG}},
\end{aligned}
$$

thus giving the NG mass parameter $M_{\mathrm{NG}}$. For full details of the derivation, we refer to Ref. [20].

\section{NUMERICAL RESULTS}

Our calculations were carried out by using the FAM-QRPA code on top of the HFB code, specifically the computer program HFBTHO [37], which is an HFB solver providing axially symmetric solutions using the harmonic-oscillator basis with the Skyrme energy density functional.

For the HFB calculations, and subsequent FAM-QRPA calculations, we have employed the Skyrme SkM* parameter set [38] at the particle-hole channel. This parameter set is know to be stable to the linear response in infinite nuclear matter [39]. The standard definition of the Skyrme EDF and associated densities can be found, e.g., in Ref. [5]. For the pairing part, the particle-particle channel interaction was taken to be a simple contact interaction without a density dependence, yielding the pairing energy density commonly called the volume pairing. In all cases studied, this type of pairing provided generally better reproduction of the data than the density-dependent mixed pairing, which in comparison yielded results lying approximately $5 \mathrm{MeV}^{-1}$ lower than those of the volume pairing. Therefore, we do not focus on the mixed pairing option in the following text. A specific nucleus in a given isotopic chain was chosen to adjust the pairing strengths separately for neutrons and protons in order to reproduce the empirical pairing gaps. Due to the zero-range pairing interaction used, it was necessary to introduce a pairing window to prevent divergent energy in the pairing channel. In this work, the quasiparticle cutoff energy was set to $60 \mathrm{MeV}$ in all cases considered. The deformed HFB ground state obtained through this setup breaks the translational, rotational, and particle number symmetries.

One of the main assets of the FAM-QRPA formalism is that no additional truncation or cutoffs of the two-quasiparticle space are imposed. This guarantees the full self-consistency between the QRPA solution and the HFB ground state. Even though the underlying HFB calculations have conserved the time-reversal symmetry, the full time-odd part of the EDF was used at the FAM-QRPA level. The FAM module implementation followed that of Ref. [21] and the FAM equations were solved in the presence of the simplex- $y$ symmetry [40].

To ensure a satisfactory convergence, we have selected 20 major oscillator shells for the HO basis in all our calculations and confirmed that no relevant improvement could be achieved from employing more shells. The FAM-QRPA iterative solution was obtained by using the very efficient modified Broyden method [41-43] that provides stable and fast convergence, especially when multiprocessor tasks are considered. Values of the spatial integrals were numerically approximated by using the Gauss-Hermite (mesh-point number set to $N_{\mathrm{GH}}=80$ ) and Gauss-Laguerre (mesh-point number set to $N_{\mathrm{GL}}=40$ ) quadratures.

In this work, we focus on two areas of the nuclear chart, the heavy rare-earth isotopes and heavy actinide isotopes. Both regions offer plenty of information about collective properties and rotational characteristics of axially deformed nuclei.

\section{A. Rotational Thouless-Valatin inertia}

To obtain the TV collective inertia we need to calculate the strength function of the symmetry restoring NG mode and take the value at zero energy (zero frequency). Since we are interested in the rotational TV inertia under the assumption of the axial symmetry and simplex- $y$ symmetry at the HFB level, we focus on the response to the $y$ component of the total-angular-momentum operator, which is a combination of the $y$ components of the orbital- and spin-angular-momentum operators $\hat{J}_{y}=\hat{L}_{y}+\hat{S}_{y}$. To investigate the role of the pairing with respect to the TV inertia, we compare the calculations where pairing correlations are taken into account with those calculations of vanishing pairing.

First, we studied the rare-earth region of the nuclear chart, specifically the erbium (even-even nuclides from ${ }^{160} \mathrm{Er}$ to ${ }^{172} \mathrm{Er}$ ) and ytterbium (even-even nuclides from ${ }^{164} \mathrm{Yb}$ to ${ }^{174} \mathrm{Yb}$ ) isotopic chains. In the case of erbium, the pairing strength parameters $\left(V_{0}^{p}=-211.20 \mathrm{MeV} \mathrm{fm}^{3}\right.$ and $V_{0}^{n}=-178.83$ $\mathrm{MeV} \mathrm{fm}^{3}$ ) were adjusted separately to ${ }^{166} \mathrm{Er}$ proton and neutron pairing gaps of $1.20 \mathrm{MeV}$ and $1.02 \mathrm{MeV}$, respectively, obtained from the three-point mass difference formula [44]. For ytterbium, we adjusted the pairing strengths $\left(V_{0}^{p}=-212.40\right.$ $\mathrm{MeV} \mathrm{fm}{ }^{3}$ and $V_{0}^{n}=-180.60 \mathrm{MeV} \mathrm{fm}^{3}$ ) to ${ }^{168} \mathrm{Yb}$ proton and neutron pairing gaps of $1.26 \mathrm{MeV}$ and $1.09 \mathrm{MeV}$, respectively.

Consequently, we calculated the TV inertia by using the same input parameters, but with the vanishing pairing option chosen (pairing strengths were selected to be negligibly small to induce a collapse of the pairing) and compared both sets of results to the experimental moments of inertia extracted from the $2^{+}$state energy of the ground-state rotational bands of studied isotopes [45]. This comparison can be found in Fig. 1.

When the volume pairing is considered, the reproduction of the experimental moment of inertia by the TV rotational inertia from FAM-QRPA is good for both erbium and ytterbium isotopic chains. It can be easily demonstrated that by adjusting the pairing strengths of all isotopes to the pairing gaps of a different nucleus from the chain, the values of the TV 

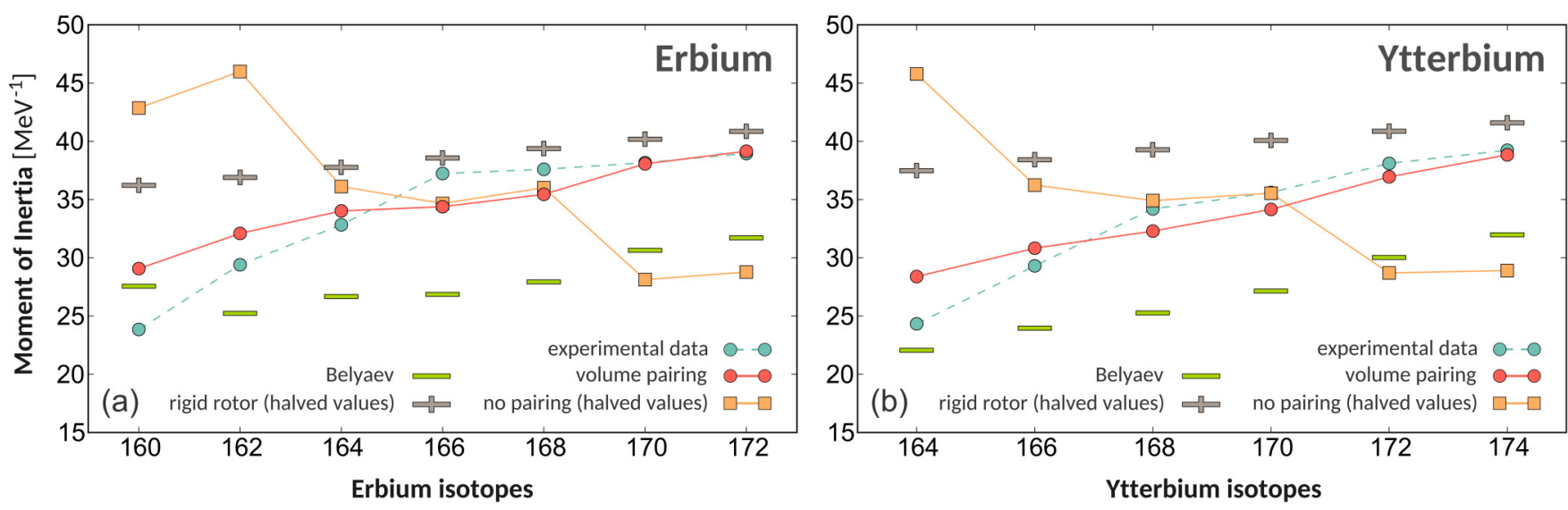

FIG. 1. Thouless-Valatin rotational moment of inertia in (a) erbium and (b) ytterbium isotopic chains. Full lines indicate the volume pairing and vanishing pairing options; in the latter case, the values of the TV inertia are divided by two in order to make a comparison with other results. The dashed lines show the experimental data from the rotational bands ( $2^{+}$states). In addition, panels show Belyaev and rigid body (divided by 2) values for the moment of inertia. For erbium isotopes, the volume pairing was adjusted to the experimental proton and neutron pairing gaps of ${ }^{166} \mathrm{Er}$, while for ytterbium isotopes, the pairing gaps of ${ }^{168} \mathrm{Yb}$ were chosen.

inertia shift up or down, basically retaining the overall pattern. Therefore, only partial improvements can be achieved by changing the pairing strengths from case to case with no significant impact on the descriptive or predictive capability.

When the nuclear pairing is not taken into account, the calculated TV inertia values are markedly higher (in Fig. 1, values without pairing are divided by two for the sake of comparison). We can conclude that the pairing correlations have crucial effects on the rotational collective motion and the TV inertia can be several times higher in contrast to the superfluid calculations.

In the next step, we focused on the actinide region of the nuclear chart, where we picked the uranium (even-even nuclides from ${ }^{232} \mathrm{U}$ to ${ }^{242} \mathrm{U}$ ) and plutonium (even-even nuclides from ${ }^{236} \mathrm{Pu}$ to ${ }^{246} \mathrm{Pu}$ ) isotopic chains. Here we again studied the effects of the pairing correlations on the rotational TV inertia and compared cases with and without pairing. The pairing strengths $\left(V_{0}^{p}=-216.18 \mathrm{MeV} \mathrm{fm}^{3}\right.$ and $V_{0}^{n}=-170.95 \mathrm{MeV} \mathrm{fm}^{3}$ ) for uranium were adjusted to the ${ }^{238} \mathrm{U}$ proton and neutron pairing gaps of $1.11 \mathrm{MeV}$ and $0.67 \mathrm{MeV}$, and the pairing strengths $\left(V_{0}^{p}=-213.20 \mathrm{MeV} \mathrm{fm}^{3}\right.$ and $\left.V_{0}^{n}=-170.80 \mathrm{MeV} \mathrm{fm}^{3}\right)$ for

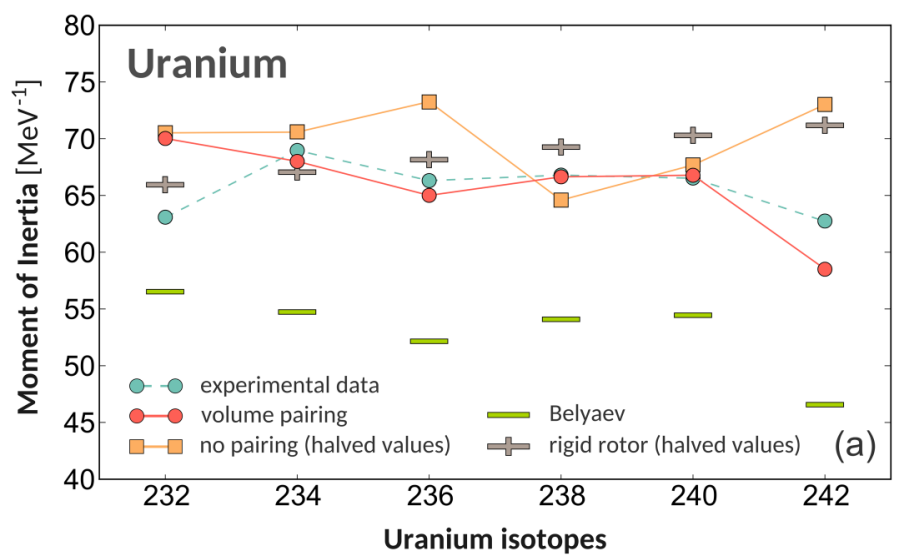

plutonium were adjusted to the ${ }^{240} \mathrm{Pu}$ proton and neutron gaps of $0.99 \mathrm{MeV}$ and $0.64 \mathrm{MeV}$, respectively. The results are shown in Fig. 2.

Once again, we obtain rather good agreement between the experiment and the rotational inertia obtained by the FAM-QRPA approach. One can see especially precise, although presumably coincidental, reproduction in the case of ${ }^{238} \mathrm{U}$ and ${ }^{240} \mathrm{Pu}$, which were the isotopes chosen for the pairing strength adjustments. The TV inertia values for collapsed pairing (values divided by two in the plots) are again notably higher, indicating the importance of the pairing.

Figures 1 and 2 also show Belyaev and rigid-body values for moment of inertia. The Belyaev moment of inertia corresponds to the approximation in which induced fields $\delta H_{\mu \nu}^{20}$ and $\delta H_{\mu \nu}^{02}$ are set to zero. For rigid-body values we used the approximation based on intrinsic deformation from Ref. [31]. The results clearly indicate that Belyaev moment of inertia underestimates the experimental moment of inertia. The magnitude of the rigid-body values is usually similar to those calculated with vanishing pairing correlations.

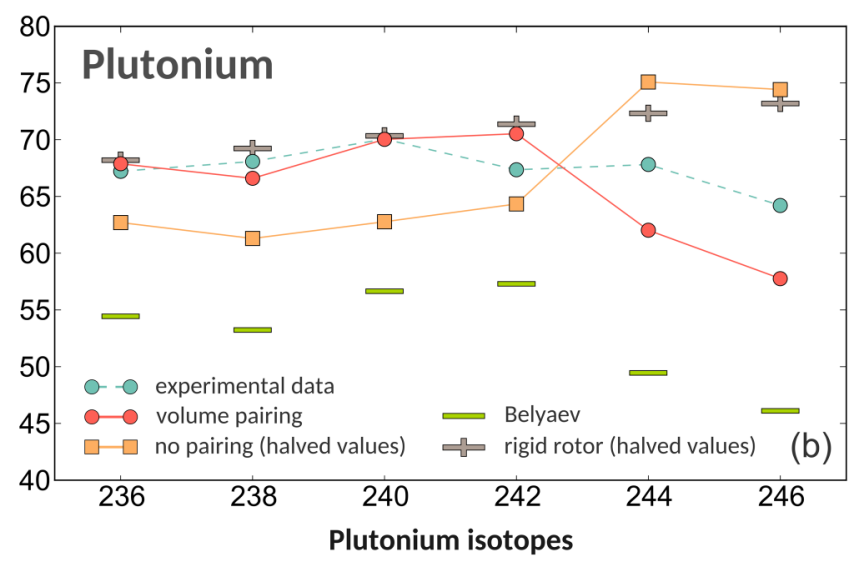

FIG. 2. The same as Fig. 1 but for (a) uranium and (b) plutonium isotopic chains. For uranium isotopes, the volume pairing was adjusted to the experimental proton and neutron pairing gaps of ${ }^{238} \mathrm{U}$, while for plutonium isotopes, the pairing gaps of ${ }^{240} \mathrm{Pu}$ were chosen. 

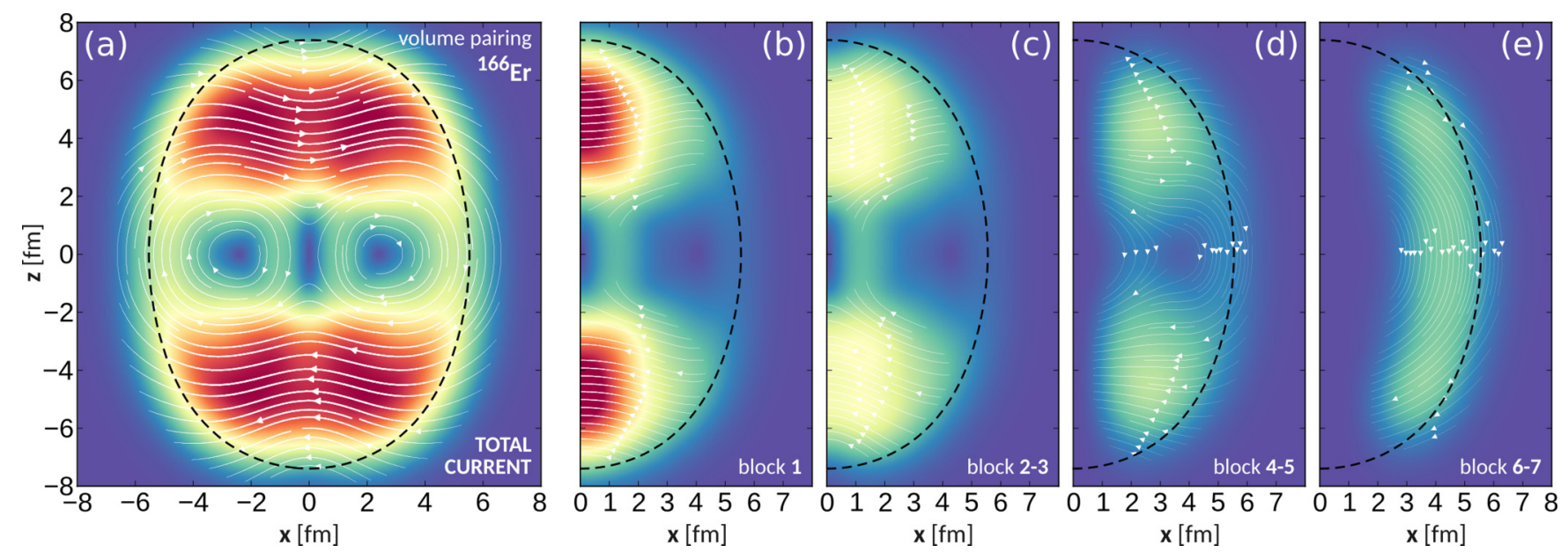

FIG. 3. The flow (lines with arrows) of the induced isoscalar current density $\vec{j}_{0}$ and its magnitude (color contours and thickness of the flow line) for ${ }^{166} \mathrm{Er}$ with volume pairing setup, obtained from the response to the $\hat{J}_{y}$ operator, are shown in the $x-z$ plane. The full cross section (a) shows the total current density, while the half plots (b)-(e) indicate the partial induced currents as given by the specific QRPA blocks. The black dashed contour line indicates the nuclear surface at matter density of $\rho_{0}=0.08 \mathrm{fm}^{-3}$. See the text for further details.

To test the precision and validity of our FAM-QRPA approach, we carried out several independent calculations by using a different HFB solver (the HFODD code [46]) and extracted the moment of inertia by using the cranking formalism. Since the HFB cranking calculations are rather time consuming, we chose 12, 14, and 16 major oscillator shells for our setup and the erbium isotope ${ }^{166} \mathrm{Er}$ as our test nucleus. These calculations were compared with the 12-, 14-, and 16-shell FAM-QRPA results. In all cases the same $\mathrm{SkM}^{*}$ parametrization was used together with identical pairing strengths.

The TV moments of inertia from the cranking formula were obtained by extrapolation to the zero cranking frequency based on the behavior of the inertia for nonzero frequencies. We obtained excellent agreement in all cases studied; for example, in the case of the 16-shell calculation, the FAM-QRPA TV inertia yields a value of $34.511 \mathrm{MeV}^{-1}$, while the cranking moment of inertia equals $34.533 \mathrm{MeV}^{-1}$. See Supplemental Material [47] for more detailed results. This excellent correspondence between the two calculations underlines the efficiency and usefulness of the FAM-QRPA formalism in calculating the rotational TV inertia.

To address the computational cost of FAM-QRPA calculations against standard cranking HFB, we estimated the time consumption of the 16-shell calculations of ${ }^{166} \mathrm{Er}$ by using an Intel Xeon 3.2 GHz CPU and compared the results from both approaches. The cranked HFB calculations using the HFODD code converged after about 4.5-10 CPU hours (depending on the chosen cranking frequency), while the HFB-QRPA calculations with the HFBTHO code took approximately 21 CPU minutes. We therefore confirm that our approach is very convenient and computationally significantly faster than standard cranking HFB calculations.

\section{B. Induced current density}

In this section, we investigate more closely the collective rotational behavior originating from the total-angularmomentum operator. As discussed previously, the NG mode, which is related to the spontaneous symmetry breaking of the rotational symmetry, leads to the TV moment of inertia connected to the strength function of the angular-momentum operator at zero frequency. Application of this operator $\left(\hat{J}_{y}\right.$ component in our case) as an external perturbation gives rise to the induced densities and currents of various types. Here we want to have a look on the induced isoscalar current density, $\vec{j}_{0} \equiv \vec{j}_{\mathrm{n}}+\vec{j}_{\mathrm{p}}$, which can give us some hints about the movement of protons and neutrons in the studied isotopes. We selected the ${ }^{166}$ Er nucleus as an example and studied the cases with and without pairing present.

In Fig. 3, one can see the $x-z$ plane cross section of the nucleus [Fig. 3(a)], where the total magnitude (color contours and line thickness) along with the directions (lines with arrows) of the total induced current density are shown. The right sides of Figs. 3(b)-3(e) show the partial contributions coming from the first seven QRPA blocks with values normalized to the same maximum value to make comparisons possible. These blocks give the largest contribution to the total TV moment of inertia. When two block numbers are indicated (e.g., block 2 and 3), there is only one plot shown since the contribution is identical from both QRPA blocks. Specifically, block 1 corresponds to quasiparticle transitions between $|\Omega|=\frac{1}{2}$ quasiparticle states. Blocks 2 and 3 correspond to transitions between $|\Omega|=\frac{1}{2}$ and $|\Omega|=\frac{3}{2}$ quasiparticle states, blocks 4 and 5 correspond to transitions between $|\Omega|=\frac{3}{2}$ and $|\Omega|=\frac{5}{2}$ states, and so on. The $\Omega$ quantum number denotes the total-angular-momentum projection of the quasiparticle state along the $z$ axis. In this way, one can understand the importance of quasiparticle states of a given $\Omega$ and their impact on the total induced current. This decomposition is rather useful when the comparison between the cases with and without pairing is made. To examine this, we calculated the induced current density and matter density under the vanishing pairing setup as well. They can be found in Fig. 4.

We already know that the presence of the pairing correlations significantly lowers the value of the rotational TV inertia 

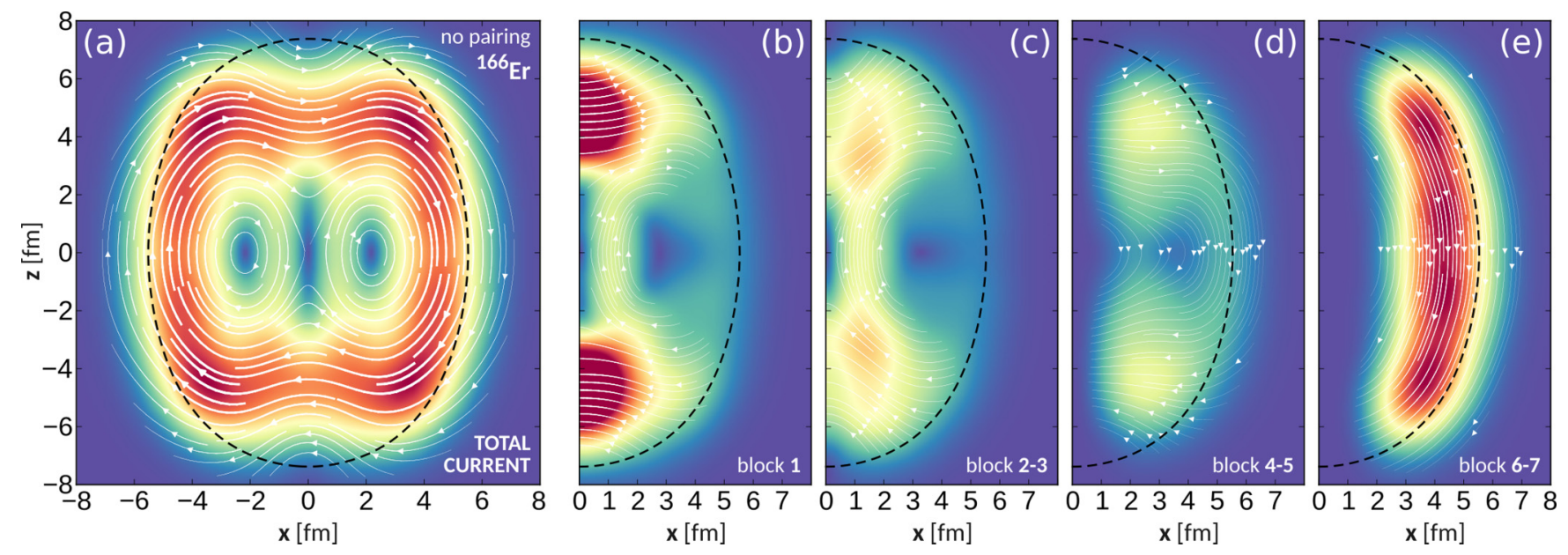

FIG. 4. Same as Fig. 3, but with vanishing pairing.

and thus one would expect to observe this effect also in the induced current density. Comparing the full plots in Figs. 3 and 4 , we see that the flow in the case with pairing resembles qualitatively more like a irrotational flow behavior, whereas the case without pairing is noticeably closer to a rigid-body rotation. However, since the moment of inertia in the case with pairing correlations included is nevertheless much higher compared with the purely irrotational case and due to the finite size of the nucleus, the shown flow pattern is not purely irrotational. Similar kinds of flow patterns also emerge with the rotation of trapped atomic gases [25], where the temperature has a critical impact on the moment of inertia and the current flow.

To track down the source of this effect, we analyzed the contributions from the QRPA blocks separately. Looking at Figs. 3(b)-3(e) and 4(b)-4(e), we see that, although the first few QRPA blocks are basically similar, a strong effect can be seen in blocks 6 and 7 in the case without pairing. The contributions from blocks 4 and 5 and especially 6 and 7 are significantly smaller when pairing is taken into account. Exactly these contributions enhance the rotational behavior in the case of vanishing pairing. Additionally, the strongest contributions (blocks 1-3) have flow patterns which are qualitatively comparable to those in Ref. [25] at low temperature. The vanishing pairing enhances the rotational component, which shifts the flow structure towards the rigid-body flow pattern. This shows that the role of high- $\Omega$ states becomes more prominent with vanishing pairing when compared with the superfluid case. Similar kind of behavior occurs in the rotational flow of ${ }^{240} \mathrm{Pu}$ and also in the flow of the oblate minimum of ${ }^{166} \mathrm{Er}$; see the Supplemental Material [47]. We did not see any large qualitative differences between the proton and neutron flow apart from the fact that the neutron flow was stronger in general due to higher abundance of neutrons.

\section{Deformation dependence of Thouless-Valatin inertia}

Rare-earth isotopes described in the present work are deformed nuclei with a moderate prolate deformation. An example of such a prolate ground state can be easily seen from the plot of the HFB binding energy versus deformation dependence of ${ }^{166} \mathrm{Er}$, as shown in Fig. 5. Here we can see that, in addition to the prolate minimum, there exists also a higherlying oblate minimum; therefore, it is interesting to examine the TV rotational inertia dependence on the deformation as well. To obtain the results for ${ }^{166} \mathrm{Er}$ seen in Fig. 5, we carried out constrained HFB calculations and scanned deformation from -0.5 to +0.5 . For each deformation point we calculated the TV inertia in the FAM-QRPA framework. As expected, the $\mathrm{TV}$ inertia vanishes in the spherical case, when the deformation is set to zero; however, once the deformation is present, it increases smoothly with the increasing deformation in both prolate and oblate cases.

One can observe small dips in the moment of inertia, which appear when the deformation is increased further away from both minima. These dips are a direct consequence of the behavior of pairing gaps and pairing energy with changing deformation. They are linked to the local increase of the pairing gaps and related increase of the pairing energy in the region on which we are focusing. Such a feature supports the general picture and conclusions about the role of the superfluidity in the properties of the rotational TV inertia.

These calculations are of great interest, since the rotational moment of inertia is one of the vital inputs for the microscopic

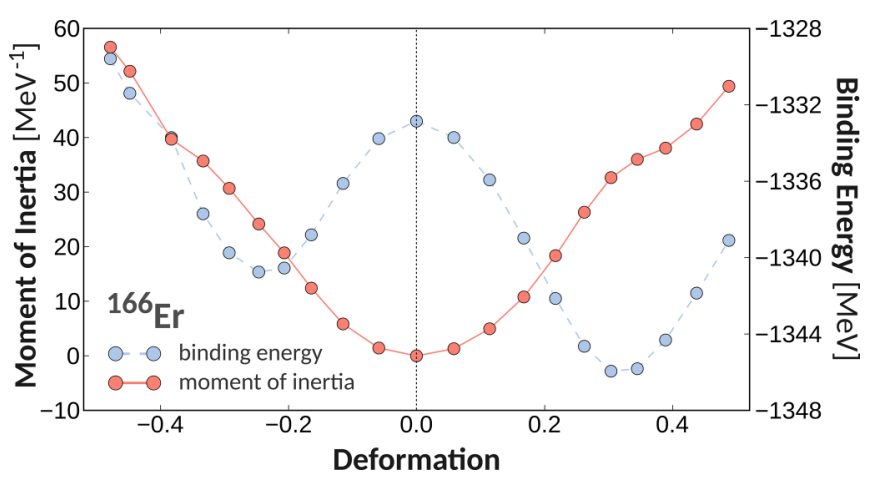

FIG. 5. Calculated Thouless-Valatin rotational moment of inertia and the HFB binding energy of ${ }^{166} \mathrm{Er}$, both as function of the deformation parameter. 
collective Hamiltonian model $[15,48,49]$ that requires such local QRPA results. Our work proves the feasibility of the FAM-QRPA for calculating collective mass parameters of this type. The FAM-QRPA framework is thus an excellent candidate for furnishing necessary quantities for this kind of models.

\section{CONCLUSION}

In the present work, we study the small-amplitude collective nuclear motion related to the rotational moment of inertia, which is a consequence of a response to the total-angularmomentum operator acting in the role of the external field. We successfully extended the FAM-QRPA approach to the case of the rotational NG mode and demonstrated the numerical efficiency in calculating important nuclear properties. This work also paves a way for the removal of the spurious component from the transition strength function, appearing with $K^{\pi}=1^{+}$type of operators when deformation is present.

Our calculations covered several axially deformed nuclei from the rare-earth and heavy-actinide regions. The analysis of the rotational Thouless-Valatin moment of inertia has shown the importance of the pairing correlations in the description of the rotational attributes of superfluid nuclei. When the pairing strength was adjusted to the experimental pairing gaps, the rotational moment of inertia for most of the isotopes was reproduced very well. The high- $\Omega$ quasiparticle states were found to have a major role in increasing the moment of inertia in the case with no pairing considered.

Lastly, we demonstrated that FAM-QRPA can be used to extract the rotational moment of inertia within the constrained HFB calculations. Such type of local QRPA calculations are essential when constructing a microscopic collective Hamiltonian model. As a future avenue, we therefore plan to employ the FAM-QRPA approach to provide such microscopic input for a collective Hamiltonian, with the purpose being to apply it to the large-amplitude collective motion in nuclei.

\section{ACKNOWLEDGMENTS}

We thank Jacek Dobaczewski, Karim Bennaceur, and Nobuo Hinohara for valuable discussions and helpful observations. This work has been supported by the University of Jyväskylä and Academy of Finland under the Centre of Excellence Program 2012-2017 (Nuclear and AcceleratorBased Physics Program at JYFL) and FIDIPRO programme. We acknowledge CSC-IT Center for Science Ltd., Finland, for the allocation of computational resources.
[1] P. Ring and P. Schuck, The Nuclear Many-Body Problem (Springer-Verlag, Berlin, 1980).

[2] M. N. Harakeh and A. van der Woude, Giant Resonances: Fundamental High-Frequency Modes of Nuclear Excitation (Oxford University Press, New York, 2001).

[3] M. Arnould, S. Goriely, and K. Takahashi, Phys. Rep. 450, 97 (2007).

[4] S. Ebata, T. Nakatsukasa, and T. Inakura, Phys. Rev. C 92, 049902(E) (2015).

[5] M. Bender, P.-H. Heenen, and P.-G. Reinhard, Rev. Mod. Phys. 75, 121 (2003).

[6] J. Erler, N. Birge, M. Kortelainen, W. Nazarewicz, E. Olsen, A. Perhac, and M. Stoitsov, Nature (London) 486, 509 (2012).

[7] A. V. Afanasjev, S. E. Agbemava, D. Ray, and P. Ring, Phys. Lett. B 726, 680 (2013).

[8] M. Kortelainen, J. McDonnell, W. Nazarewicz, E. Olsen, P.G. Reinhard, J. Sarich, N. Schunck, S. M. Wild, D. Davesne, J. Erler, and A. Pastore, Phys. Rev. C 89, 054314 (2014).

[9] J. Terasaki and J. Engel, Phys. Rev. C 82, 034326 (2010).

[10] J. Terasaki and J. Engel, Phys. Rev. C 84, 014332 (2011).

[11] S. Péru, G. Gosselin, M. Martini, M. Dupuis, S. Hilaire, and J.-C. Devaux, Phys. Rev. C 83, 014314 (2011).

[12] M. Martini, S. Péru, and M. Dupuis, Phys. Rev. C 83, 034309 (2011).

[13] T. Nakatsukasa, T. Inakura, and K. Yabana, Phys. Rev. C 76, 024318 (2007).

[14] P. Avogadro and T. Nakatsukasa, Phys. Rev. C 84, 014314 (2011).

[15] T. Nakatsukasa, K. Matsuyanagi, M. Matsuo, and K. Yabana, Rev. Mod. Phys. 88, 045004 (2016).

[16] M. Stoitsov, M. Kortelainen, T. Nakatsukasa, C. Losa, and W. Nazarewicz, Phys. Rev. C 84, 041305 (2011).
[17] N. Hinohara, M. Kortelainen, and W. Nazarewicz, Phys. Rev. C 87, 064309 (2013).

[18] T. Nikšić, N. Kralj, T. Tutiš, D. Vretenar, and P. Ring, Phys. Rev. C 88, 044327 (2013).

[19] M. T. Mustonen, T. Shafer, Z. Zenginerler, and J. Engel, Phys. Rev. C 90, 024308 (2014).

[20] N. Hinohara, Phys. Rev. C 92, 034321 (2015).

[21] M. Kortelainen, N. Hinohara, and W. Nazarewicz, Phys. Rev. C 92, 051302(R) (2015).

[22] T. Oishi, M. Kortelainen, and N. Hinohara, Phys. Rev. C 93, 034329 (2016).

[23] Kai Wang, M. Kortelainen, and J. C. Pei, Phys. Rev. C 96, 031301(R) (2017).

[24] M. Farine, P. Schuck, and X. Viñas, Phys. Rev. A 62, 013608 (2000).

[25] M. Urban and P. Schuck, Phys. Rev. A 67, 033611 (2003).

[26] M. Urban, Phys. Rev. A 71, 033611 (2005).

[27] M. W. Zwierlein, J. R. Abo-Shaeer, A. Schirotzek, C. H. Schunck, and W. Ketterle, Nature (London) 435, 1047 (2005).

[28] I. Bausmerth, A. Recati, and S. Stringari, Phys. Rev. A 78, 063603 (2008).

[29] S. Riedl, E. R. Sánchez Guajardo, C. Kohstall, J. Hecker Denschlag, and R. Grimm, New J. Phys. 13, 035003 (2011).

[30] A. Bulgac, Annu. Rev. Nucl. Part. Sci. 63, 97 (2013).

[31] A. Bohr and B. R. Motelson Nuclear Structure (Benjamin, New York, 1975), Vol. II.

[32] D. J. Thouless and J. G. Valatin, Nucl. Phys. 31, 211 (1962).

[33] W. Satuła, J. Dobaczewski, W. Nazarewicz, and M. Rafalski, Phys. Rev. Lett. 106, 132502 (2011).

[34] Y. Nambu, Phys. Rev. 117, 648 (1960).

[35] J. Goldstone, Nuovo Cimento 19, 154 (1961).

[36] N. Hinohara and W. Nazarewicz, Phys. Rev. Lett. 116, 152502 (2016). 
[37] M. Stoitsov, N. Schunck, M. Kortelainen, N. Michel, H. Nam, E. Olsen, J. Sarich, and S. Wild, Comput. Phys. Commun. 184, 1592 (2013).

[38] J. Bartel, P. Quentin, M. Brack, C. Guet, and H.-B. Håkansson, Nucl. Phys. A 386, 79 (1982).

[39] A. Pastore, D. Davesne, Y. Lallouet, M. Martini, K. Bennaceur, and J. Meyer, Phys. Rev. C 85, 054317 (2012).

[40] J. Dobaczewski, J. Dudek, S. G. Rohoziński, and T. R. Werner, Phys. Rev. C 62, 014310 (2000).

[41] C. G. Broyden, Math. Comp. 19, 577 (1965).

[42] D. D. Johnson, Phys. Rev. B 38, 12807 (1988).

[43] A. Baran, A. Bulgac, M. M. Forbes, G. Hagen, W. Nazarewicz, N. Schunck, and M. V. Stoitsov, Phys. Rev. C 78, 014318 (2008).
[44] G. F. Bertsch, C. A. Bertulani, W. Nazarewicz, N. Schunck, and M. V. Stoitsov, Phys. Rev. C 79, 034306 (2009).

[45] IAEA-Nuclear Data Services, Evaluated Nuclear Structure Data File, https://www-nds.iaea.org

[46] N. Schunck, J. Dobaczewski, J. McDonnell, W. Satuła, J. A. Sheikh, A. Staszczak, M. Stoitsov, and P. Toivanen, Comput. Phys. Commun. 183, 166 (2012).

[47] See Supplemental Material at http://link.aps.org/supplemental/ 10.1103/PhysRevC.97.034321 for additional calculated results.

[48] S. G. Rohoziński, J. Phys. G 39, 095104 (2012).

[49] K. Matsuyanagi, M. Matsuo, T. Nakatsukasa, K. Yoshida, N. Hinohara, and K. Sato, Phys. Scr. 91, 063014 (2016). 